\title{
Personen- und Werkregister
}

Abeles, Otto 521

Abraham, Felix 509

Abraham, Ulf 348

Abramowitsch, Scholem-Jankew 375

Achad Haam 107, 110, 384

Acher, Mathias Siehe Birnbaum, Nathan

Ackermann, Aron

Die Schwergeprüften 444

Adler, Bill 448

Adler, Friedrich 297, 330, 336

Adler, H[ans] G[ünther] 226-227

Auschwitz 226-227

Adler, Hans G. 286

Adler, Jeremy 227

Adler, Paul 443-444

Die Zauberflöte 443

Elohim 443

Glauben aus unserer Zeit 443

Über das Judentum 436

Adler, Viktor 299

Adorno, Theodor W. 201, 223-224, 226-227, 274, 562

Kulturkritik und Gesellschaft 223

Meditationen zur Metaphysik 223

Minima Moralia 223

Negative Dialektik 223

Noten zur Literatur 223

Agnon, Samuel Josef 419, 547, 549, 551

Agnon, Shai 208

Aguilar, Grace

The Vale of Cedars 394

Aichinger, Helga Michie 236

Aichinger, Ilse 236

Aufruf zum Mißtrauen 236

Das Fenster-Theater 237

Die größere Hoffnung 236

Verschenkter Rat 237

Alcharisi, Jehuda

Tachkemoni 470

Alexis, Willibald 88, 95, 409

Allan, Seán 575

Allmendinger, Karl

Shylock unter Bauern 28

Alon, Alexander 463-478

Aloni, Jenny 206, 212, 215
Das Brachland 212

Zypressen zerbrechen nicht 212

Altenberg, Peter 298, 315

Altendorf, Wolfgang

Thomas Adamsohn 493

Altermann, Nathan 212

Altman, Mosche (Moses) 366

Altmann, Alexander 45

Améry, Jean 233

Hand an sich legen 233

Jenseits von Schuld und Sühne 199

Amichai, Jehuda 205

Amir, Dov 209-211

Anders, Günther 226, 233-234

Mensch ohne Welt 234

Andersch, Alfred

Die Rote 30

Efraim 30

Sansibar oder der letzte Grund 30

Angress, Ruth K. Siehe Klüger, Ruth

Anonymus

Artushof 18

Anonymus

Artussage 15

Anonymus

Dukus Horant 15-16

Anonymus

Jüngeres Hildebrandtslied 16

Anonymus

Ma'asse-Buch 17, 383

Anonymus

Paris un Wienne 17

Anonymus

Schalschelet ha-Kabala 472

Anonymus

Schewet Jehuda 472

Anonymus

Schmuel-Buch 16

Anonymus

Sigenot 16

Anonymus

Tristan 15

Anonymus

Widuwilt 15, 17

Antiochus 114 
Anton, Karl, Steinhoff, Hans und Maisch, Herbert Ohm Krüger (Film) 568

Antonescu, Ion 366, 371

Apitz, Bruno

Nackt unter Wölfen 30

Apollinaire, Guillaume 277

Appelfeld, Aharon 369

Aptroot, Marion 11

Arendt, Hannah 63, 191, 226, 233, 284, 498

Eichmann in Jerusalem 226

Argov, Sascha 212

Aristophanes 90-91

Arnim, Achim von $41,87-88,480$

Halle und Jerusalem 26, 479-480

Arno, Siegfried 567

Arns, Alfons 569

Fatale Korrespondenzen 565

Arnstein, Nathan 66

Artmann, Hans Carl 237

Asch, Sholem 376, 383-385

Ascher, Saul

Leviathan 58

Asher, Adolf 542

Ashkenazi, Ofer 567

Asser, Rose 272

Atze, Marcel 227

Aub, Joseph 518

Auerbach, Bernard 398

Auerbach, Berthold 78, 392, 394-395, 504

Briefe an Jakob Auerbach 410

Dichter und Kaufmann 55, 392, 409

Schwarzwälder Dorfgeschichten 409

Spinoza 27, 392-393, 409-410

Auerbach, Jakob 518

Ausländer, Ignaz 231

Ausländer, Rose 229, 231-232, 365-368, 370-371

36 Gerechte 232

Bis an den Nagelmond 232

Der Regenbogen 231, 367

Rückblick 232

Ausubel, Nathan 448

Avenarius, Ferdinand 124, 127128, 130 Aussprache mit Juden 129

Avi-Yona, Eva 213

Avni, Stella 368

Bach, Johann Sebastian 65

Bachofen, Johann Jakob 143
Backhaus, Fritz 565

Badeni, Graf Kasimir Felix 331

Baeck, Leo 508

Bahr, Erhard 196

Bahr, Hermann 306, 308, 310

Bailan, Zhu Siehe Blum, Klara

Baioni, Giuliano 349

Báky, Josef von

Der Ruf 570

Balcke, Ernst 284

Ball-Hennings, Emmy Ruf und Echo 284

Balzac, Honoré de 40

Bamberger, Johann Peter 60-61

Baretzki, Stefan 365

Bartels, Adolf 108, 455 Heinrich Heine 456 Judentum und deutsche Literatur 79 Kritik und Kritikaster. Mit einem Anhang: Das Judentum in der deutschen Literatur 79

Bartov, Omer 565, 569

Basil, Otto 366

Der Plan 236

Basnage, Jacques 92

Basnizki, Eva 213

Bathdori, Sulamith 208

Bato, Ludwig 521

Battegay, Caspar 270-280, 575

Battenberg, Friedrich 11

Bauer, Bruno Die Judenfrage 99,139

Bauer, Felice 113, 346-347

Bauer, Yehuda 227-228

Bauer,Markus 196

Baum, Oskar 111, 118, 208, 275, 328, 335 Das Volk des harten Schlafes 355 Die böse Unschuld 441

Baumgardt, David 283 Erinnerungen an Georg Heym 289

Baumgarten, Jean 11

Baumgarten, S. J. Joseph von Eichendorff 454

Bayerdörfer, Hans-Peter 167

Bebenburg, Karg von 306

Becher, Johannes R. 284-285

Beck, Karl

Saul 482

Becker, Ben 575

Becker, Julius 509 
Becker, Jurek 253

Bronsteins Kinder 573

Beckett, Samuel

Warten auf Godot 492

Beer, Johann 41

Beer, Michael 78

Beer-Hofmann, Richard 118, 298, 305, 307, 316-318

Der Graf von Charolais 316, 483

Der Tod Georgs 317

Die Historie von König David 484

Jáakobs Traum 307

Schlaflied für Mirjam 318, 344

Beethoven, Ludwig van 66, 130

Behr, Isachar Falkensohn 45, 52-55, 78, 270

Gedichte von einem pohlnischen Juden 53 , $59,270-271$

Behrens, Katja 255

Behrens, Leffmann 12

Beller, Steven 300-304

Ben Abraham, Jakob

Maassebuch 379

Ben Amos, Dan 448, 465

Ben-Chorin, Schalom 164, 180, 202, 208, 211212, 437

Bruder Jesus. Der Nazarener in jüdischer

Sicht 212

Das Messiasspiel 444

Ben-Chorin, Schalom und Stern, Gerson Menora 201, 207-208

Ben-Chorin, Schalom und Chajes, Adolf Die Ernte 203, 208

Bendavid, Lazarus 52, 56, 271

Benedikt, Moriz 309-310

Ben-Gavriêl, Moshe Ya'akov 206, 211

Das Haus in der Karpfengasse 211

Frieden und Krieg des Bürgers Mahaschavi 211

Kleines Palästinabuch für empfindsame

Reisende 211

BenGershom, Ezra

Der Esel des Propheten 448

Ben-Gurion, David

Jiskor - Ein Buch des Gedenkens an gefallene Wächter und Arbeiter im Lande Israel 377

Benigni, Roberto

La Vita è Bella (Film) 570
Benjamin, Walter 122, 152, 274, 286, 438-439,

474-475, 562

Beim Bau der chinesischen Mauer 475

Ursprung des deutschen Trauerspiels 561

Versuch eines Schemas zu Kafka 474

Benvenisti, J. L. 298

Ben-Yitzchaky, Saul 208

Benyoëtz, Elazar 214, 232

Benz, Wolfgang 167

Beradt, Martin 164

Berdyczewski Siehe Bin Gorion, Micha Josef

Bergelson, David 381, 419

Berger, Peter L. 448

Bergius, Hanne 456

Bergmann, Hugo 111, 208, 330, 338

Bergner, Elisabeth 486, 492

Berliner, Abraham 13

Bérlioz, Hector 274

Bermann-Fischer, Gottfried 402

Bernays, Michael 558

Bernstein, Aron 78, 415

Vögele, der Maggid 415

Best, Otto F. 11

Bettauer, Hugo 302

Die Stadt ohne Juden 302, 568

Beyer, Frank

Jakob der Lügner 570

Bialik, Chaim Nachman 103, 107, 117, 383, 547

Das hebräische Buch 103

Biermann, Wolf 253

Biller, Maxim 246-248, 254-255, 257, 262, 279,432

Der gebrauchte Jude 246-247, 432

Die Tempojahre 247

Die Tochter 257

Harlem Holocaust 247

Im Kopf von Bruno Schulz 279

Wenn ich einmal reich und tot bin 279

Bin Gorion, Micha Josef 464

Philo-Lexikon 551-552

Binder, Hartmut 334

Birch-Pfeiffer, Charlotte 26

Birnbaum, Menachem 116-117

Birnbaum, Nathan 111, 116, 126, 276

Achad Haam 103

Den Ostjuden ihr Recht! 114

Selbst-Emanzipation 101

Zur Frage des jüdischen Geisteslebens in

Deutschland 126 
Nationale Wiedergeburt des jüdischen

Volkes in seinem Lande 101

Birnbaum, Salomo A. 376-377

Birnbaum, Uriel

In Gottes Krieg 160

Bismarck, Otto von 452

Blanchot, Maurice 231

Blass, Ernst 283-284, 291

Blass, Ernst

Alfred Kerr 290

Blau, Armin 440-441

Bloch, Ernst 177, 191

Bloch, Hans 509

Blok, Aleksandr 277

Blum, Klara 367-368, 385

Blum, Robert, Herloßsohn, Carl und Marggraf, Hermann

Allgemeines Theater-Lexikon 27

Blumenberg, Hans 238

Blumenberg, Ursula 238

Blumenfeld, Kurt

Erlebte Judenfrage 132

Blumenthal, Hermann 416, 418

Blumenthal, Oscar 86-87

Blumenthal-Weiss, Ilse 164, 180

Bluwstein, Rachel 208

Boas, C. 540, 542

Bock, Tobias 50

Bodemann, Y. Michal 244, 262-263

Bodenheimer, Alfred 270-280, 438-439

Bodenheimer, Max 208

Boeckh, August 71

Boesche-Zacharow, Tilly 210

Boese, Carl

Keine Feier ohne Meyer 567

Böhm, Adolf 547

Böhmer, Roland 15

Boleslav, Netti 212

Bollack, Jean 229

Vor dem Gericht der Toten 229

Bonnet, Charles

Philosophische Untersuchung der Beweise

für das Christentum 49

Bormann, Alexander von 457

Börne, Ludwig 78, 84-86, 89-91, 137-138, 274, 449-451, 481

Briefe aus Paris 85-86, 88, 93, 95, 137

Der Esskünstler 95

Menzel der Franzosenfresser 88, 95
Monographie der deutschen Postschnecke 95

Bosch, Roselyne

La Rafle 570

Bourget, Paul 40

Boyarin, Daniel 197

Boyarin, Jonathan 197

Boysen, Friedrich Eberhard

Koran-Übersetzung 137

Brahm, Otto 484

Brandeis (Verlag) 545

Brandeis, Jakob B. 397

Brandeis, Jakob W. 520

Brandeis, Richard 520

Brandes, Georg 558

Brann, Markus 508

Brasch, Thomas

Der Passagier 570

Braschwitz, Baruch 541

Braun, Helmut 232

Brecht, Bertolt

Furcht und Elend des Dritten Reiches 497

Mutter Courage 496

Brenner, Michael 83

Brentano, Clemens 87-88

Die Schachtel mit der Friedenspuppe 26

Gockel, Hinkel und Gackeleia 26

Victoria und ihre Geschwister 26

Brentano, Franz 334

Breslauer, Hans Karl

Die Stadt ohne Juden (Film) 567

Breuer, Isaak 191

Breuer, Mordechai 56

Breysach, Barbara 276

Březina, Otokar 336

Hymnen 336

Briegel, Manfred 194

Brinckmann, Karl Gustav 64

Broch, Hermann 225, 312

Die Schlafwandler 225

Die Schuldlosen 225

Brod, Max 111, 118, 208, 211, 275, 284, 326$328,335,337-338,344-345$

Arnold Beer 111, 344

Der jüdische Dichter deutscher Zunge 111 Heidentum, Christentum, Judentum 338 Jüdinnen 111, 328

Rëubeni, Fürst der Juden 355, 402-403, 440-441

Streitbares Leben 122, 326 
Tycho Brahes Weg zu Gott 326, 338, 341,

$$
\text { 402-403 }
$$

Unambo 211

Unsere Literaten und die Gemeinschaft 113, 115

Broder, Henryk M. 458

Volk und Wahn 457-458

Brody, Heinrich 508

Bronner, Gerhard 448

Bruckner, Ferdinand

Die Verbrecher 487

Elisabeth von England 487

Krankheit der Jugend 487

Bruners, Wilhelm 210, 213

Brunner, Constantin 100, 367

Buber, Martin 102-103, 105, 107, 110-112, 118, $128,146,171,177-178,180,183,191,209$,

211-212, 214, 231, 332, 344-345, 375-379,

382, 435, 439, 484, 511, 544-545, 549, 551

Das jüdische Prag 111

Der Jude 140, 546, 548

Die Losung 114-115

Die Mächtigkeit des Geistes 183

Die Tempelweihe Siehe Die Losung

Drei Reden über das Judentum 111, 129 , 338, 344, 436

Geschehende Geschichte 183

Jüdische Künstler 105

Jüdische Renaissance 435

Tora-Übersetzung 136

Unser Bildungsziel 183

Buber, Salomon 463

Bubis, Ignatz 244

Bucher, Lothar 86

Bucherer, Wilhelmine 211

Büchner, Georg 366

Woyzeck 26

Buck, Theo 229

Bukofzer, Werner 208, 213

Bun, Alexandru cel 362

Burg, Josef 366

Burg, Meno 428-429

Geschichte meines Dienstlebens 428

Busch, Isidor 517-519

Büschenthal, Lippmann Moses 78, 516

Byron, Lord 90

Caesar, Gaius Julius 131

Caligula 487
Calvary, S. (Verlag) 540, 542

Campe, Joachim Heinrich 531

Campe, Julius 96

Canetti, Elias 225, 255, 308, 316, 352

Die Blendung 308

Masse und Macht 225, 316

Carlebach, Joseph

Der neue Glaubensbegriff als Grundlage

der Bibelübersetzung von Buber

Rosenzweig 438

Carlson, Marvin 494

Carrington, Herbert 23

Caspari, Hedwig Salomos Abfall 444

Cassel, David Geschichte der jüdischen Literatur 76, 138

Cassirer Bruno 544

Cassirer, Ernst 191

Cassirer (Salon) 290

Centralverein deutscher Staatsbürger jüdischen Glaubens

Anti-Anti. Tatsachen zur Judenfrage 548

Celan, Paul 191, 196, 212, 223, 227-231, 233 , 245, 274, 277-278, 365-366, 368-371, 440, 559

Die Niemandsrose 278

Engführung 230

Todesfuge 193, 229, 367-368

Zürich, Zum Storchen 232

Céline, Louis-Ferdinand 40

Chajim Hameln 426

Chajes, Adolf und Ben-Chorin, Schalom Die Ernte 203, 208

Chamberlain, Houston Stewart 455

Chamizer, Raphael 521

Chargaff, Erwin 370

Charleville, Esther de 61

Chase, Jefferson S. 89, 449, 451

Chaucer, Geoffrey 40

Chomsky, Marvin J. Holocaust 496, 571, 573

Claudius, Matthias 179

Claus, Horst 565

Cohen, Elieser Lippmann Siehe Behrens, Leffmann

Cohen, Hermann 100, 140, 191

Cohen, Sarah Blacher 448

Cohen, Tobias 47

Cohn, Emil Bernhard 437, 522 
Cohn, Erich 116

Corbea-Hoişie, Andrei 369

Cosmas von Prag

Chronica Boemorum 327

Croneburg, Benjamin 501

Cummings, E[dward] E[stlin] 232, 367

Curtius, Julius

Der lebende und dennoch maustodte M.G.

Saphir 450

Czerski, Alexander 213

Dahm, Volker 167, 539

Dalberg, Karl Theodor von 84

Danquart, Didi

Viehjud Levi 573

Danton, George 285

Daudet, Alphonse 40

Davidson, Hans Siehe Van Hoddis, Jakob

De Lemos, Benjamin 61

De Man, Paul 423

Debussy, Claude 284

Degen, Michael 496

Deleuze, Gilles 209

Denkler, Horst 23

Deppisch, August 454

Derrida, Jacques

Feu la cendre 231

Kraft der Trauer 231

Schibboleth 231

A Self-Unsealing Poetic Text 231

Dessauer, Adolf 314

Großstadtjuden 314

Deutsch, Ernst 489, 492

Devidels, Mariane 63

Diamant, Jacob

Es waren Kosaken im Städtelein 155

Frei 155

Israel im Kriege 155, 159

Krieg 156

Dickens, Charles 37, 40-41, 349

Dickinson, Emily 277

Diderot, Denis 62

Diner, Dan 1, 244-245, 262, 571

Dinesen, Ruth 167

Dinter, Artur

Die Sünde wider das Blut 28

Dinur, Benzion 45

Dische, Irene 250-252, 260

Fromme Lügen 251
Dischereit, Esther 255

Distelmeyer, Jan 565

Distl, Egon Siehe Goldstein, Moritz

Döblin, Alfred 118, 141, 276, 456

Babylonische Wandrung 147

Der deutsche Maskenball 456

Reise in Polen 276

Dohm, Christian Wilhelm

Über die bürgerliche Verbesserung der Juden 83

Dohm, Ernst 87

Dohna-Schlobitten, Friedrich Ferdinand Alexander zu 64

Dohna-Schlobitten, Friedrich Karl Emil zu 64

Domin, Hilde 232

Nur eine Rose als Stütze 232

Donath, Adolph 103, 105, 107

Dössekker, Bruno Siehe Wilkomirski, Binjamin

Drach, Albert 221, 224, 226, 369, 457

Das große Protokoll gegen

Zwetschkenbaum 225

Unsentimentale Reise 226

Dräger, Heinrich 238

Dreifuß, Alfred 492

Dressen, Wulf Otto 17

Drewitz, Ingeborg

Alle Tore waren bewacht 492

Dreyfus, Alfred 488

Droller, David 546

Drozdowski, Georg 369

Drumont, Edouard

La france juive 100

Dubno, Salomon 51

Dubnow, Simon 141, 547

Dühring, Eugen 100, 291, 455

Die Judenfrage als Racen- Sitten- und

Culturfrage 139

Dujardin, Éduard 310

Les lauriers sont coupés 310

Durian, Wolf 536

Durieux, Tilla 284

Eberle, Joseph 455

Ebner-Eschenbach, Marie von 28

Edelmann-Ohler, Eva 151-163

Edschmid, Kasimir

Das Bücher-Dekameron 291

Egger, Urs

Epsteins Nacht 574 
Ehrenstein, Albert 141, 191, 193, 315

Ansichten eines Exterritorialen 141

Menschlichkeit 142

Zion 142

Ehrmann, Daniel 464

Ehrmann, Herz 401

Eichendorff, Joseph von 454-455

Eichmann, Adolf 226, 493

Einstein, Carl 456

Der blutige Ernst 456

Eisenmenger, Johann Andreas 300

Entdecktes Judentum 300

Eisner, Pavel 333

Elbogen, Ismar 379, 508

Eldad der Daniter 469

Eliasberg, Alexander 381-382

Elijaschew, Isidor 376

Eliot, T[homas] S[tearns] 40

Eloesser, Arthur 164

Erinnerungen eines Berliner Juden 173-174

Vom Ghetto nach Europa 270

Eminescu, Mihai 366

Engel, Erich

Affaire Blum 570

Engels, Friedrich

Deutscher Sozialismus in Versen und Prosa 85

Enoch, Samuel 504, 506

Ephraim, Veitel Heine 54

Erpenbeck, Jenny

Aller Tage Abend 31

Heimsuchung 31

Ersch, Johann Samuel und Gruber, Johann

Gottfried

Allgemeine Enzcyklopädie 74

Escher, Karl 164, 169-170

Der Sohn des großen Königs 181

Eskeles, Bernhard 66

Esway, Alexander

Hercules Maier 567

Ettighofer, Paul Coelestin

Mädchen ohne Stern 30

Ettlinger, Jakob Aaron 505

Euchel, Isaac Abraham 48, 52, 58, 501

Eybenberg, Marianne von 64

Faerber, Meir 204

Auf dem Weg 206, 210

Fanta, Bertha 334
Färberböck, Max

Aimée und Jaguar (Film) 572

Farbstein, Moses 106

Fassbinder, Rainer Werner 244

Der Müll, die Stadt und der Tod 31, 42, 244

Faurisson, Robert 224

Feilchenfeld, Alfred

Denkwürdigkeiten der Glückel von Hameln 379

Feinberg-Jütte, Anat 491-499

Feiner, Shmuel 45-46

Feingold, Marko 223

Feiwel, Berthold 103, 105, 107, 110, 521, 545

Jüdischer Almanach auf das Jahr 5663105

Fest, Joachim 31

Fetting, Hugo 457

Feuchtwanger, Lion 141, 144, 402-404, 481

Der historische Prozeß der Juden 144

Der jüdische Krieg 144

Der Tag wird kommen 402

Die Jüdin von Toledo 403-404

Die Söhne 402

Die Geschwister Oppermann 571

Jud Süß 402-403, 487, 569

Nationalismus und Judentum 144

Spanische Ballade Siehe Die Jüdin von Toledo

Fiala-Fürst, Ingeborg 337

Fichman, Lejser 369

Field, Nathanael 317

Field, Nathanael und Massinger, Philip The fatal dowry 317

Fischer, Torben 24

Fischhof, Adolf 296

Flake, Otto

Die großen Worte 290

Fleischel, Egon 544

Fleischmann, Lea 213, 253

Flusser, Vilém 222

Fock, Gustav 543

Fontane, Theodor 28, 41

Der Stechlin 28

Die Poggenpuhls 28

Effi Briest 28

L'Adultera 28

Unwiederbringlich 28

Formstecher, Salomon 518

Förster-Nietzsche, Elisabeth 285

Fraenkel, David 502 
Francolm, Isaac Assur Siehe Rispart, Eugen

Frank, Hans 260

Frank, Niklas 260

Der Vater 260

Frank, Rudolf 164,169

Ahnen und Enkel 172, 181

Fränkel, David 49

Fränkel, Maimon 516

Frankel, Zacharias 505-508

Frankenberg, Sigmund 517

Frankfurter-Wolf, Ulla 415

Frankl, Ludwig August 78, 398, 464

Frankl, Pinkus Friedrich 508

Franz Ferdinand (Erzherzog von Österreich) 312

Franz Joseph I. (Kaiser von Österreich) 301, 303, 363

Franzos, Karl Emil 366, 416, 504

Der Pojaz 364, 416

Die Juden von Barnow 416

Nach dem höheren Gesetz 441

Freimann, Aron 508, 515

Frenzel, Elisabeth 23

Freud, Sigmund 143, 255, 260, 297-299, 305, 308, 310-311, 313, 497, 555, 561-562

Der Mann Moses und die monotheistische Religion 308

Der Witz und seine Beziehung zum

Unbewußten 449

Selbstdarstellung 299

Traumdeutung 308

Zur Psychopathologie des Alltagslebens 308

Freudenthal, Gideon 56

Freudenthal, Max 508

Freytag, Gustav 37

Soll und Haben 27-28, 41

Fried, Babette 413

Fried, Erich 194, 457

Friedenthal, Herbert Siehe auch Freeden,

Herbert

Der Klassentag 174

Die unsichtbare Kette 172-173

Leben zur falschen Zeit 172

Friedlaender, Salomo 284

Friedländer, David 48, 52, 58, 60, 66, 516, 541

Lesebuch für jüdische Kinder 530-531

Friedländer, Moses 66

Friedländer, Saul 167, 227

Friedmann, Louis 312

Friedrich II. von Preußen 55, 61
Frisch, Efraim 544

Zenobi 437

Frisch, Max

Andorra 494

Fritsch, Theodor

Handbuch der Judenfrage 122, 454

Fritz, Susanne 336

Frohberg, Regina 66

Schmerz der Liebe 66

Frost, Robert 277

Fruchtmann, Benno 213

Frühwald, Wolfgang 87, 177, 194

Fuchs, Rudolf 208, 336

Fuks, Laijb 16

Fürnberg, Louis 204

Fürst, Julius 503, 507

Fürstenthal, Ernst

Abraham 171, 181

Fürstenthal, Raphael 464, 518

G(h)uttmann, Wilhelm Simon 283

Gadamer, Hans Georg 180

Gaius Julius Caesar Siehe Caesar, Gaius Julius

Galinski, Heinz 496

Gallwitz, Tim 570

Gans, David 325

Gans, Eduard 70, 92, 453

Ganzfried, Daniel 256, 261

Der Absender 255-256

Geiger, Abraham 503, 506, 518

Jüdische Zeitschriften 506

Geiger, Ludwig 15, 23, 80, 109, 504, 507, 558

Berlin 1688-1840 454

Der Kunstwart und die Judenfrage 126

Die Juden und die deutsche Literatur 79

Gelber, Mark H. 27, 37-44

Gellert, Christian Fürchtegott

Leben der Schwedischen Gräfin von $G * * 25$

Gellhaus, Axel 231

Geoffroy, Louis de 86

George, Stefan 558

Gershom ben Jehuda, Rabbi 9

Gerstl, Elfriede 221, 237

Gillman, Abigail 305

Gilman, Sander 3

Ginsberg, Ascher Siehe Achad Haam

Glaeser, Ernst

Jahrgang 190229

Glagau, Otto 100 
Glasenapp, Gabriele von 391-406, 407-421, 514-526, 527-538, 539

Glikl von Hameln Die Memoiren der Glückel von Hameln 12, 379-380, 426

Goebbels, Joseph 169 Der Angriff 458

Goes, Albrecht Das Brandopfer 30

Goethe, Johann Wolfgang von $41,53,56,72-74$, 89-91, 130, 148, 179, 339, 453, 479, 481

Die Leiden des jungen Werther 558 Dichtung und Wahrheit 425

Faust 486, 497

Iphigenie auf Tauris 5

Italienische Reise 1, 5

[Rezension] Behr, Gedichte eines pohlnischen Juden 53

Weltliteratur 2

Goetschel, Willi 274, 473

Goldberg, Lea 208

Goldberg, Oskar 283, 286-287, 289

Die fünf Bücher Mosis ein Zahlengebäude 283

Golde, Louis 546

Goldmann, Nachum 116

Goldmark, Josef 296

Goldschlag, George A. Gedichte 180

Goldschmidt, Georges-Arthur 194

Goldschmidt, Lazarus 549

Der Talmud 552

Goldschmidt, Meir Aron 418

Goldschmidt, Victor 552

Goldstein, Franz 208

Goldstein, Julius 127 Moritz Goldsteins ,Deutsch-jüdischer Parnaß' 127

Goldstein, Moritz 107-110, 118, 122-132

Aesthetik und Kunstwerk 131

Begriff und Programm einer jüdischen

Nationalliteratur 122-123, 126, 130

Berliner Jahre 131

Deutsch-jüdischer Parnaß 79, 90, 107, 122-132, 127, 132

Führers Must Fall 131

Geistige Organisation des Judentums 107, 123, 130

German Jewry’s Dilemma 122, 127, 130
Über das Wesen des Judentums 123

Goldstein, Walter 208

Goldstücker, Eduard 333

Goll, Claire 229, 368

Goll, Yvan 141, 229

Hiob 193

Jean-sans-Terre 144

Goltschnigg, Dietmar 455

Gombrich, Ernst H. 3

Gompertz, Theodor 299

Gomperz, Ruben Elias 12

Goncourt, Edmond de 40

Gong, Alfred 368

Goodrich, Frances und Hackett, Albert Das Tagebuch der Anne Frank 493

Gordin, Jacob 381

Gordon, Jehuda Leib 58

Gorelik, Lena 264, 278-279

Hochzeit in Jerusalem 264

Lieber Mischa 264

Meine weißen Nächte 264

Sie können aber gut Deutsch 279

Gorionides, Josephus

Josippon 469

Goßens, Peter 230

Gottgetreu, Erich 213

Gottschall, Rudolf von

Die deutsche Nationalliteratur des

neunzehnten Jahrhunderts 453-454

Grab, Walter 456

Grabbe, Christian Dietrich Aschenbrödel 481

Graetz, Heinrich 505, 508, 543

Graf, Dominik

Im Angesicht des Verbrechens 575

Grass, Günter 30-31, 41

Beim Häuten der Zwiebel 43

Die Blechtrommel 30

Hundejahre 30

Was gesagt werden muss 43

Grathoff, Dirk 458

Greene, Graham 40

Greiner, Bernhard 438-439, 476

Grigorjewittsch, Gabriel Siehe Behr, Isachar Falkensohn

Grillparzer, Franz 481

Die Jüdin von Toledo 27, 482

Esther 482

Grimm, Jacob und Wilhelm 466 
Deutsches Wörterbuch 449, 454

Märchen 532

Grimmelshausen, Hans Jakob Christoffel von $18,24,41$

Gronemann, Sammy 117, 201, 208, 212, 547

Der Weise und der Narr 212

Erinnerungen eines Jecken 212

Hawdoloh und Zapfenstreich 159

Schalet, Beiträge zur Philosophie des

„Wenn schon“ 448

Zu meiner Entlastung 207

Gross, Otto 143

Grossman, Jeffrey 435, 474

Grotthus, Sophie Leopoldine Wilhelmine von 64

Grözinger, Karl Erich 348

Gruber, Johann Gottfried und Ersch, Johann

Samuel

Allgemeine Enzcyklopädie 74

Grünbaum, Herbert 492, 496

Grundig, Lea 202

Grundmann, Regina 472

Grünfeld, Max 413

Gruschka, Roland 11, 375-387

Guattari, Felix 209

Gubser, Martin 27

Güdemann, Moritz 13

Gumpertz, Aaron 56, 271

Gundolf, Friedrich 558

Günsburg, Karl Siegfried 516

Gutsche, Victoria 23-36

Gutu, George 370

Gutzkow, Karl 88, 94, 96

König Saul 481

Uriel Acosta 481

Haacke, Wilhelm 455

Hackett, Albert und Goodrich, Frances

Das Tagebuch der Anne Frank 493

Haecker, Hans Joachim

Dreht Euch nicht um 493

Hagen, Heinrich von der

Neueste Wanderungen, Umtriebe und

Abenteuer des Ewigen Juden 451-452

Hahn, Barbara 66

Hainz, Martin A. 221-243, 229-230, 362-374

Hakel, Hermann 448

Halkin, Schmuel 385

Halle-Wolfssohn, Aaron 52
Halpern, Ben

Exile 198

Hambrock, Matthias 539-554

Hamburger, Michael 194

Hameiri, Avigdor 208, 211

Hammer, Almuth 439

Händel, Georg Friedrich 481

Hannibal 308

Harap, Louis 448

Harden, Maximilian 86, 309, 457

Hardenberg, Karl August von 130

Harlan, Veit Jud Süß (Film) 494, 565, 568-569

Harz, Benjamin 540, 542, 547, 549

Hauff, Wilhelm 409 Jud Süß 27, 569

Lichtenstein 393

Haug, Wolfgang 456

Hauschner, Auguste 275

Der Tod des Löwen 341

Die Familie Lowositz 330

Haverkamp, Alfred 14

Hebbel, Christian Friedrich

Der Diamant 482

Herodes und Mariamne 482

Judith 27, 482-483

Hebel, Frieda 213

Hebel, Johann Peter 382

Hegel, Georg Wilhelm Friedrich 71

Hegner, Jakob 544, 549

Hehn, Viktor 454

Gedanken über Goethe 453

Heidegger, Martin 226, 229-230, 559

Heidenheim, Wolf Benjamin 541

Sidur Sefat Emet 542

Heiman, Shlomo ben Josua 271

Heimann, Moritz 544

Heine, Heinrich 59, 77-78, 86, 88-90, 99, 137138, 140, 145, 149, 182, 202, 255, 273-274, $309,315,369,394-395,448-450,454$, 458, 463-464, 472-473, 475, 481, 547

Almansor 481, 497

Briefe aus Berlin 86, 95

Briefe aus Helgoland 94

Der Rabbi von Bacherach 92, 392, 473-474

Die Bäder von Lucca 89, 450

Die romantische Schule 95, 274

Französische Zustände 85, 274

Gedichte 454 
Geständnisse aus der Matzratzengruft 92, 138

Hebräische Melodien 473

Jehuda ben Halevy 473

Ludwig Börne 93-95, 137-138

Lutetia $86,274,473$

Reisebilder 273, 454

Romanzero 92-93, 473

Salon III 88

Shakespeares Mädchen und Frauen 274

Über den Denunzianten 88

Vermischte Schriften 274

Zur Geschichte der Religion und

Philosophie in Deutschland 274

Heinemann, Isaak 508

Heinemann, Jeremias 516

Heinrich-Jost, Ingrid 456

Helbig, Heinz

Leinen aus Irland (Film) 568

Hellberg, Frank 456

Heller, B. (Verlag) 546

Heller, Isidor 414

Herder, Johann Gottfried 72, 74, 123, 148

Die älteste Urkunde des

Menschengeschlechts 176

Herlitz, Georg und Kirschner, Bruno

Jüdisches Lexikon 1, 549, 552

Herloßsohn, Carl, Blum, Robert und Marggraf, Hermann

Allgemeines Theater-Lexikon 27

Herman, Mark

The Boy in the Striped Pyjamas (Film) 570

Hermand, Jost 89

Hermann, H. S. 546

Hermann, Matthias 437

Herrmann, Hugo 111, 509

Herrmann, Leo 111, 509

Herrnfeld, Anton (Theater) 566-567

Herrnfeld, Donat (Theater) 566-567

Hertz, Deborah 67

Herz, Henriette 61, 63-65, 88

Herz, Marcus 52, 61, 63-64, 541

Herzberg, Isaak 401, 415

Herzberg-Fränkel, Leo 416-417

Polnische Juden 416

Herzig, Arno 12, 84

Herzl, Theodor 103, 110, 131, 190, 207, 297-

$299,305,307,313,317,319-320,510$
Altneuland 102, 110, 319

Das neue Ghetto 299, 319

Der Judenstaat 101-102, 114, 299, 320

Der Mann Moses und die monotheistische

Religion 319

Die Juden Prags zwischen den Nationen 332

Das lenkbare Luftschiff 102

Herzog, Hillary Hope 297, 305

Herzog, Wilhelm

Die Affäre Dreyfus 488

Politik und Literatur 288

Heß, Mendel 503

Hess, Moses 190

Hessel, Franz 164

Alter Mann 174

Hessing, Jakob 213, 244-269

Heuberger, Rachel 500-514

Hevesi, Simon 522

Heym, Georg 283-285, 289

Der Krieg 285, 292

Zu den Wahlen 288

Heymann, Fritz

Der Chevalier von Geldern 402

Hickl, Max 521

Hilberg, Raul 227

The Destruction of the European Jews 227

Hildesheimer, Esriel 506

Hildesheimer, Hirsch 506

Hildesheimer, Wolfgang 196, 204, 208, 227 ,

253, 277

The End of Fiction 227

Hilfrich, Carola 51

Hiller, Kurt 113, 283-285, 288, 290, 292

Französlinge 288-290

Hilsenrath, Edgar 204, 235-236, 369, 457-458

Bronskys Geständnis 236

Das Märchen vom letzten Gedanken 235

Der Nazi \& der Friseur 236, 245

Fuck America 235

Nacht 236, 245

Hilsner, Leopold 331

Himmler, Heinrich 497

Hinkel, Hans 168-170, 550

Hippel, Theodor Gottlieb von Lebensläufe nach aufsteigender Linie 25

Hippler, Fritz

Der ewige Jude (Film) 568

Hirsch, Karl Jakob 437, 444

Hochzeitsmarsch in Moll 444 
Hirsch, Leo 164, 169

Botschaft aus Granada 404

Die Letzte Station 172, 181

Gespräch im Nebel 402, 404

Hirsch, Samson Raphael 202, 399-402, 437, 444, 505, 543

Hirsch, Sara 505

Hirschbein, Peretz 381

Hirschbiegel, Oliver

Ein ganz gewöhnlicher Jude (Film) 575

Hirschel, Moses 54

Hirschfeld, Werner 209

Hitler, Adolf 122, 131-132, 236, 247, 250, 253, $257,260-261,301,371,494,497-498$

Hochhuth, Rolf

Der Stellvertreter 493

Hochwälder, Fritz 494

Der Himbeerpflücker 494

Hoddis, Jakob van

(Nebbich auf jüdisch) 289

Hödl, Sabine 12

Hoeflich, Eugen Siehe Ben-Gavriêl, Moshe

Ya'akov

Hoffmann, Daniel 435-447

Hoffmann, E[rnst] T[heodor] A[madeus] 340

Die Brautwahl 24, 26

Hofmann, A. J. (Verlag) 542, 552

Hofmann, Martha 208

Hofmannsthal, Hugo von 113, 128, 284, 287, 306, 312

Brief an Karg von Bebenburg 306

Ein Brief (Chandos-Brief) , 286, 312

Das kleine Welttheater 444

Der Dichter und diese Zeit 286

Hofschtejn, Dovid 385

Holdenried, Michaela 422

Hölderlin, Friedrich 284, 559

Holdheim, Samuel 299, 503

Holitscher, Arthur

Der Golem 445

Holland, Agnieszka

Hitlerjunge Salomon (Film) 571

Hollander, Jacob 506

Homberg, Herz 51

Honecker, Erich 263

Honigmann, Barbara 149, 253-255, 260, 262, 264, 278

Damals, dann und danach 255

Roman von einem Kinde 254, 278
Honigmann, David 396, 518

Horch, Hans Otto 1-6, 10, 23, 27, 167-168, 196, $210,391,500-514$

Hostivít 325

Houwald, Ernst von 26

Howard, John A. 11

Höxter, John 117

Humboldt, Alexander von $63,65,83$

Humboldt, Wilhelm von $63,65,556$

Hurwicz, Elias 377

Hurwitz, Heimann (Hyman)

Sagen der Hebräer 467-469, 471, 475

Imhoof, Markus

Das Boot ist voll (Film) 571

Immermann, Karl Leberecht 89

Die Epigonen 27

Inquit Siehe Goldstein, Moritz

Isaak ben Mordechai 325

Isler, Meyer 518

Israel ben Elieser, Rabbi Siehe Baalschem

Itzig, Cäcilie 66

Itzig, Daniel 65-66

Itzig, Fanny 66

Itzig, Moritz 480

Itzig, Sara 65-66

Itzkowski, H. 546

Jacob, Paul Walter 492

Jacobsohn, Wilhelm 542

Jacoby, Joel 78

Jaeger, Achim 9-23

Jagow, Bettina von 439

Jamme, Christoph

Martin Heidegger 229

Janik, Allan 305

Janik, Allan und Toulmin, Stephen

Wittgensteins Wien 305

Janin, Jules 88

Janowitz, Franz 334

Janowitz, Hans 334

Jean Paul 91, 95, 382, 451

Jelinek, Elfriede 221

Jelinek, Friedrich 221

Jellinek, Adolf 463

Jenninger, Philipp 244

Jesenská, Milena 331, 344

Jessner, Leopold 489, 491

Jesus Christus 38-39, 311, 355, 436 
Joachimsthal-Schwabe, Anna

Gedichte 180

Jodl, Friedrich 331

Johann von Luxemburg 325

Johnson, William M. 303

Jokl, Anna Maria 213

Begegnung am Toten Meer 213

Jolowicz, Leo 543

Josel ben Gershon von Rosheim 12

Joseph II. (Kaiser) 83, 297, 326, 330

Josephus, Flavius 403, 482

Jost, Isaak Markus 518

Allgemeine Geschichte des Israelitischen

Volkes 391, 504

Judas Makkabäus 155

Jung, Johann Heinrich 427

Jungmann, Max 116-117

Kadmon, Stella 204

Kafka, Franz 111, 113, 118, 151, 209, 221, 234, $275,289,326-328,330-332,334-335$, $337,339,344-346,351,367,438-439$, 463-464, 472, 474-475, 549, 551

Aphorismen 349, 351

Beschreibung eines Kampfes 326

Brief an den Vater 343

Das Stadtwappen 327, 330, 332

Das Urteil 113, 345-346

Der Heizer 338

Der Hungerkünstler 347

Der Process 347, 350, 475

Der Verschollene 338, 476

Die Brücke 345

Die Verwandlung 113, 350

Ein Bericht für eine Akademie 345

Eine Kreuzung 333

Einleitungsvortrag über Jargon 275, 346

Fahrt zur Mutter 350

Gemeinschaft 335

Schakale und Araber 332, 343

Tagebücher 350

Vor dem Gesetz 347-348, 350, 475

Kahler, Erich von

Israel unter den Völkern 146

Kahn, Arthur 415

Kaindl, Raimund Friedrich 366

Kaléko, Mascha 164, 179-180, 204, 213, 277

Kleines Lesebuch für Große 174

Lyrisches Stenogrammheft 174, 213
Kalisch, David 85, 87, 90, 452, 483

Einer von unsere Leut' 483

Einmalhunderttausend Thaler 87

Kaminer, Wladimir 263

Russendisko 263-264

Kanner-Schildberger, Hertha

Hadassa 180

Kant, Immanuel 55, 60-61, 63, 130, 467

Kanter, Daniel Christoph 541

Kapper, Siegfried 330, 414

Kara, Avigdor 325

Karasek, Hellmuth 457, 493

Karl Alexander von Württemberg 13, 569

Karl der Große 17

Karpeles, Gustav 504, 506

Geschichte der Jüdischen Literatur 2, 77, 139

Karsten, Dietrich Ludwig Gustav 63

Käsler, Dirk 560

Kastein, Josef 208, 211

Eine Palästinensische Novelle 211

Kasten, Jürgen 565

Kästner, Erich 536

Katz, Albert 504

Katz, Friedrich Siehe Kettner, Friedrich

Katz, Jacob 13, 45

Katzenelson, Jizchak 253

Kauffmann, Isaak 540, 542-544, 546, 552

Kaufmann, David 508

Kaufmann, Fritz Mordechai 378, 547

Kaufmann, Moses Wolf 540, 542, 544

Käutner, Helmut In jenen Tagen (Film) 570

Kawalerowicz, Jerzy Bronsteins Kinder (Film) 573

Kaznelson, Siegmund 303, 331, 549, 552 Juden im deutschen Kulturbereich 3, 552

Keilson, Hans 164, 169

Kenig Artis Hof Siehe Anonymus Artushof

Keren, Else 213

Kernmayer, Hildegard 86

Kerr, Alfred 85-86, 290-291, 457-458, 483

Kertész, Imre 458

Fiasko 222

Ich - ein anderer 222

Kaddisch für ein nicht geborenes Kind 222

Liquidation 222

Roman eines Schicksallosen 222

Kettner, Friedrich 367 
Kilcher, Andreas B. 3, 70-82, 99-121, 135-150, 253, 255, 424, 457

Kipphardt, Heinar Joel Brand 493

Kirchheim, Raphael 518

Kirschner, Bruno und Herlitz, Georg Jüdisches Lexikon 1, 549, 552

Kisch, Egon Erwin 234, 329-330

Kisch, Matthias 398, 414

Kishon, Ephraim 448

Kittner, Alfred 229, 368

Klabund 369

Klaghofer, Wolfgang 439

Klapp, Michael 397, 414

Klaproth, Martin Heinrich 63

Klatzkin, Jakob 116, 139-140

Grundlagen des Nationaljudentums 140

Klausner, Margot 208, 213

Klein, Karl 517-519

Kleinert, Andreas und Wessel, Kai Klemperer - Ein Leben in Deutschland (Film) 573

Kleist, Heinrich von 64

Klemperer, Victor 168 Ich will Zeugnis ablegen bis zum letzten 224, 573

LTI 224

Kley, Eduard 518

Klimt, Gustav 310 Nuda Veritas 310

Klötzel, Cheskel Zwi 109, 132, 547 Das grosse Hassen 132 Deutsch-jüdischer Parnaß 132

Klötzel, Hans 509

Kluge, Alexander

Abschied von gestern 571

Klüger, Ruth 30, 234-235, 431-432, 458 weiter leben 235, 432

Knapp, Fritz Peter 16

Köbner, Wilhelm 543

Koepke, Wulf 196

Kohn, Abraham 518

Kohn, Hans 111, 118, 303

Kohn, Salomon 396-398, 414

Der Kadisch vor Col-Nidre in der Altneu-

Synagoge 397

Der Retter 397

Gawriel 397, 399

Koller, Werner 377
Kolmar, Gertrud 164, 167, 174-176, 179, 181, 193-194

Das Wort der Stummen 175

Der Engel im Walde 175

Die Frau und die Tiere 176

Die Tiere von Ninive 174

German Sea 175

Möblierte Dame 172

Susanna 174,181

Wir Juden 175, 183

Kompert, Leopold 78, 411-412, 504 Aus dem Ghetto 411

Konfuzius 315

König, René 560

Königsberger, Annemarie 213

Kornfeld, Paul 334

Die Verführung 485 Jud Süss 488

Körte, Mona 23

Kortner, Fritz 485, 488, 492-493, 570

Die Zwiesprache 492

Donauwellen 492

Kossak, Ernst 86

Kracauer, Siegfried 191, 561-562

Krafft, Carl 467, 471-472 Jüdische Sagen und Dichtungen nach den Talmuden und Midraschen 470

Kraft, Werner 212, 430 Worte aus der Leere 212

Kraiker, Gerhard 458

Krämer, Markus 522

Kramer, Stanley Judgment at Nuremberg (Film) 573

Kraus, Karl 112, 299, 305, 308-310, 315, 320, 367, 449, 453, 458

Das Bild des Siegers 310

Die Fackel 366

Die letzten Tage der Menschheit 309, 456, 485

Eine Krone für Zion 320

Er is doch ä Jud 309

Harakiri und Feuilleton 309

Heine und die Folgen 315

Schicksal der Silbe 315

Kraus, Oskar 334

Krechl, Ursula Landgericht 196

Kreimeier, Klaus 565, 568

Kreindler, Leon 513 
Krochmal, Nachman 463

Krojanker, Gustav 116-118, 547 Juden in der deutschen Literatur 117

Kronberg, Simon 437, 442-443 Chamlam 443

Kruntorad, Paul 498

Kugelmann, Cilly 565

Kuh, Anton 141 Juden und Deutsche 143

Kuh, David 334

Kuh, Ephraim Moses 45, 52, 54-55, 78, 393, 409

Der polnische Jude, der ein Christ ward 55

Kühn, Siegfried

Die Schauspielerin 571

Kühn-Ludewig, Maria 539

Kühne, Jan 201-220

Kulke, Eduard 413

Kunth, Gottlob Johann Christian 63

Kürnberger, Ferdinand 86

Kwitko, Lejb 385

Lajarrige, Jacques 369

Lamm, Louis 540, 542-543, 546, 548

Jüdische Kriegsliteratur 158

Landau, Leo 17

Landau, Lola 213

Landmann, Salcia 11

Der jüdische Witz 448

Jüdische Witze 448

Landsberger, Arthur 124 Judentaufen 124

Lang, Isaac Siehe Goll, Yvan

Langenhorst, Georg 437

Langer, Felicia 213

Langer, Georg 111

Lasker-Schüler, Else 107, 110, 117-118, 191, 193, 203-204, 208, 211, 245, 284, 439

Arthur Aronymus und seine Väter 489

Der Prinz von Theben 110

Die Nächte der Tino von Bagdad 110

Die Wupper 485

Hebräische Balladen 110

Laube, Heinrich 94, 451

Lauer, Gerhard 458

Lavater, Johann Caspar 49-50

Lavoisier, Antoine Laurent de 63

Lazare, Bernard 191

Lazarus, Moritz 2, 559-560
Le Rider, Jacques 305

Lehmann, Jürgen 231

Lehmann, Marcus 399-400, 402-403, 505, 520

Aus Vergangenheit und Gegenwart 400

Die Familie y Aguillar 400

Eine Sedernacht in Madrid 400

Ester Chiera 400

Lehmann, Oscar 400, 505

Lehnardt, Andreas 463

Lehrberger, M. 541-542, 544, 552

Leibniz, Gottfried Wilhelm 442

Leiwik, Halpern

Der Gefangene 171

Lejeune, Philippe 423

Leppin, Paul 333, 340

Das Gespenst der Judenstadt 340

Severin 328

Lessing, Gotthold Ephraim 49, 54, 90-91, 95 , $130,148,271,289,479$

Die Juden 24-25, 479

Nathan der Weise 25, 479, 491-492

Lessing, Theodor

Jüdischer Selbsthass 449

Lessmann, Max 546

Letteris, Meir 464

Levi, Primo 222

Ist das ein Mensch? 222

Levin Varnhagen, Rahel 65-66, 88, 272-273, 453, 480

Rahel. Ein Buch des Andenkens an ihre

Freunde 272-273

Levin, Schmarja 106

Lévinas, Emmanuel 231

Levita, Elia

Bovo-Buch 17

Levy Rabbin, Cerf Isaac 426

Levy, Berthold 546

Levy, Dani

Alles auf Zucker! (Film) 575

Mein Führer (Film) 498

Meschugge (Film) 574

Levy, Samuel Salomon 65

Levy, Sarah 480

Levy, Walter 208

Lewinsky, Charles

Ein ganz gewöhnlicher Jude (Film) 575

Melnitz 255

Lichtenberg, Georg Christoph 468

Lichtwitz, Max 546 
Liebeneiner, Wolfgang

Bismarck (Film) 568

Die Entlassung (Film) 568

Liebermann, Max 128

Liebknecht, Karl 141

Liman, Friedrika Siehe Marcuse, Friederike

Liepmann, Abraham Nathan 65

Liepmannssohn, Selig Louis 517

Lilien, Ephraim Moses 103-104, 107, 522, 545, 552

Limor, Micha

Der Traum 207

Lind, Jakov 457

Lindau, Paul 86-88

Lindtberg, Leopold 489

Lipman, Steve

Laughter in Hell 448

Lissauer, Ernst 80, 99, 109, 135, 209

[Sprechsaal] 127

Haßgesang gegen England 127

Livay, Yvonne 213

Liwa ben Bezalel, Jehuda Siehe Löw, Rabbi

Lobe, Friedrich 204, 492

Locke, John 49

Locke, Thomas 50

Loening, Carl Friedrich 544, 549

Loerke, Oskar 179

Loeven, Alfred

Philo-Lexikon 551-552

Loewe, Heinrich 101, 106, 117

Die Sprachen der Juden 107

Eine jüdische Nationalbibliothek 107

Feinde ringsum! 114

Loewenberg, Jakob 109

[Sprechsaal] 127-128

Loewenson, Erwin 283-285, 287-289

Die Décadence der Zeit und der „Aufruf“

des „Neuen Clubs“ 286-288, 290

Druidenwacht 287

Neopathos 287

Löwenstein, Hilde Siehe Domin, Hilde

Loewenthal, Alwin 547

Loewy, Ernst 167, 195-196

Loghin, Constantin 370

Lombroso, Cesare 455

Loos, Adolf 310

Lorenz, Otto 223

Lorz, Andrea 539

Louis Ferdinand von Preußen 65

Louis Philippe I. 85, 93
Löw, Rabbi 325, 338, 342-343, 403

Löwenstein, Fritz 509

Löwenstein, Rudolf 87

Lowenstein, Steven M. 457

Löwit, R. 540, 542, 546-547, 549

Löwy, Jizchak 275, 345-348, 350

Lubitsch, Ernst 565-567, 575

Meyer aus Berlin (Film) 567

Schuhpalast Pinkus (Film) 566-567

Lueger, Karl 301, 303, 306, 455

Lühe, Irmela von der 196

Lukács, Georg 475

Lumet, Sidney

The Pawnbroker (Film) 570

Lustiger, Arno 249

Lustiger, Gila 249-250, 262

Die Bestandsaufnahme 249-250, 256

So sind wir 256

Luther, Martin 41, 90-91

Von den Juden und ihren Lügen 12

Luxemburg, Rosa 141

Luzatto, Samuel David 503

Lyotard, Jean-François 224

Der Widerstreit 224, 226

M., H.

[Sprechsaal] 128

Ma'asse-Buch Siehe Anonymus, Ma'asse-Buch

Maetzig, Kurt

Ehe im Schatten (Film) 570

Magris, Claudio 337

Mahler, Gustav 299, 449

Mahomet 137

Maien, Carl 517

Maimon, Salomon 46, 52, 55-57, 255, 271-272, 427

Lebensgeschichte 56, 59, 271-272, 427

Maimonides, Moses 49, 55, 57, 272, 530

More Nevuchim 49, 55, 272

Maisch, Herbert, Steinhoff, Hans und Anton, Karl

Ohm Krüger (Film) 568

Malo, Markus 422-434

Mandelstam, Ossip 231, 277-278

Manger, Itzig 366

Mann, Daniel und Sargent, Joseph Playing for Time (Film) 570

Mann, Heinrich 42

Der Untertan 29 
Die Göttinnen 29

Die Schauspielerin 29

Fulvia 29

Geist und Tat 288

Im Schlaraffenland 29

Mann, Thomas 24, 29, 41-42, 279

Brief aus Deutschland 291

Buddenbrooks 29

Gladius Dei 29

Königliche Hoheit 29

Tristan 29

Wälsungenblut 29

Mannheim, Karl 560

Mapu, Abraham 58

Marcus, M. \& H. (Verlag) 543, 552

Marcus, Max 543

Marcus, Theodor 552

Marcuse, Friederike (Fradchen) 65

Marggraf, Hermann, Blum, Robert und

Herloßsohn, Carl

Allgemeines Theater-Lexikon 27

Margulies, Heinrich

Das Galuthproblem im Zionismus 140

Margul-Sperber, Alfred 229, 366-367, 371

Der unsichtbare Chor 370

Die Buche 370

Markisch, Peretz 419

Marlowe, Christopher 40

Marr, Wilhelm 100

Der Sieg des Judenthums über das Germanenthum 452

Martin, Karl Heinz 485

Marty, Anton 334

Marx, Hilde 164, 209

Marx, Julius

Kriegs-Tagebuch eines Juden 158

Marx-Peters, Hilde Im Vers gefangen 180

Massinger, Philip 317

Massinger, Philip und Field, Nathanael The fatal dowry 317

Mayer, Hans 23

Mayer, Paul 176, 179

Mayer, Ruth 197

Mayer, Samuel 517

Meerbaum Eisinger, Selma 369

Mehler, Frieda

Vom Wege 171

Mehring, Sabine von 575
Mehring, Walter 456

Der Kaufmann von Berlin 488

Meidner, Ludwig 164

Meisl, Mordechai 325

Menasse, Eva 260

Vienna 257-258

Menasse, Hans 257-258

Menasse, Robert 258

Die Vertreibung aus der Hölle 404, 457

Mendele Moicher Sforim 419, 448, 547

Mendelssohn, Abraham 65

Mendelssohn, Fromet 63

Mendelssohn, Joseph 530

Mendelssohn, Moses 45-46, 48-50, 57-58, 63-65, 202, 254, 270-271, 289, 384, 474, 479, 501, 518, 530, 541

Bibelübersetzung 10, 408

Bi'ur-Übersetzung 52, 529-530

Jerusalem 50, 74

Phädon 49-50

Philosophische Gespräche 49

Über die Empfindungen 49

Mendelssohn-Bartholdy, Felix 65

Mendes, Lothar Jew Süss 569

Menkes, Hermann 416, 418

Menzel, Wolfgang 88-90, 95, 451, 455

Deutsche Vierteljahrsschrift 451

Die deutsche Literatur 90

Ludwig Börne, Briefe aus Paris I 450

Mertens, Leo 180

Metternich, Klemens Wenzel Lothar von 274

Meyer, Mariane 64

Meyer, Sara 64

Meyer, Seligmann 506

Meyer, Uriel 208

Meyerbeer, Giacomo 99, 449, 481

Meyerowitz, Jan 448-449

Meyer-Sickendiek, Burkhard 83-98, 448-462

Meyrink, Gustav 327, 339-342

Der Golem 339-340

Michaelis, Mirjam 213

Michaelis, Paul 114

Mieses, Isaac 416

Miething, Christoph 425, 432

Mihaileanu, Radu

Train de vie 570

Milder, Anna 65

Miron, Dan 437 
Mischel, Salome 370

Mittelmann, Hanni 189-200

Mombert, Alfred

Aeon 484

Mommsen, Theodor 283

Monasch, Baer Loew 540, 542

Monk, Egon

Die Bertinis (Film) 571

Die Geschwister Oppermann (Film) 571

Moore, Marianne 232, 367

Morgenstern, Matthias 439

Morgenstern, Soma 277

Die Blutsäule 277

Moritz, Karl Philipp 56, 271, 427

Andreas Hartknopf 25

Mosenthal, Salomon von Deborah 483

Moser, Moses 70, 92

Moses 305, 308, 319, 348

Moses, Julius 464

Jüdischer Novellenschatz 545

Moses, Margarete 208

Diesen und auch Jenen hat Gott gemacht 204

Mosse, Rudolf 87

Moszkowski, Alexander 448

Motzan, Peter 370

Mozart, Wolfgang Amadeus

Die Zauberflöte 443

Müller, Adam 88

Müller, Georg 544

Münchhausen, Börries von

Die neue Dichtung 291

Mundt, Theodor 94

Munk, Eduard 518

Muschg, Walter 456

Mynona Siehe Friedlaender, Salomo

Nadel, Arno 107, 164, 179

Das Leben des Dichters 180

Nadler, Josef

Literaturgeschichte des deutschen Volkes 458

Napoleon Bonaparte 83-85, 123, 272, 480

Naschitz, Fritz 213

Nathenson, Cary 575

Natonek, Hans 326-327

Kinder einer Stadt 326, 328, 338

Nekula, Marek 275

Neruda, Jan 336
Nerval, Gérard de 274

Nestroy, Johann 87

Judith und Holofernes 483

Neuburger, Otto

Philo-Lexikon 551-552

Neuman, Oscar 213

Neumann, Alfred 544

Neumann, Angelo 329

Neumann, Robert 457

An den Wassern von Babylon 148

Neumann, Ronnith 261

Nicolai, Friedrich 541

Leben und Meinungen des Herrn Magister

Sebaldus Nothanker 25

Niehoff, Maren 463, 474

Nietzsche, Friedrich 93, 142-143, 284-286, 353

Der Wille zur Macht 285

Zarathustra 140

Nistor, Ion 370

Nolden, Thomas 249-250, 278

Noll, Chaim 148, 213, 253-254, 262, 264

Noll, Dieter 253

Noll, Hans Siehe Noll, Chaim

Nordau, Max 104

Nossig, Alfred 107

Moderne jüdische Dichtung 105

Proba rozwiazania kwestji zydowskiej 105

Novak, William 448

Novalis (Friedrich von Hardenberg) 468

Och, Gunnar 23-36, 449-450

Olsvanger, Immanuel

L'Chayim! 448

Oppenheimer, Franz 510

Oppenheimer, Johann F.

Philo-Lexikon 551-552

Oppenheimer, Joseph Süß 13, 487

Oppenheimer, Samuel 12

Osmomysl, Jaroslaw 362

Osten, Michael Siehe Goldstein, Moritz

Otokar II., Přemysl 325

Otten, Karl 141

Pagis, Dan 205

Pakula, Alan J.

Sophie's Choice (Film) 570

Palm, Hilde Siehe Domin, Hilde

Papavassiliou, Jorgo

Liebe unter Verdacht (Film) 574 
Pappenheim, Bertha 377, 379-380

Allerlei Geschichten 380

Gebete 180

Zeenah u Reenah 380

Pascheles, Alfred 519

Pascheles, Jakob 397, 519

Pascheles, Jakob W.

Sippurim 396

Pascheles, Samuel 519

Pascheles, Wolf 396-397, 464, 519-520, 542

Pavell, Lilit 213

Pazi, Margarita 335

Perel, Solomon 571

Perez, Jizchak Leib 375, 381-382, 419, 435, 448, 547

Bontsche Schwajg 376

Perutz, Leo 204, 341-343, 404

Nachts unter der steinernen Brücke 341, 403-404

Pfemfert, Franz 141, 283

Die Aktion 142

Jüngste tschechische Lyrik 336

Pfizer, Gustav

Heine's Schriften und Tendenz 451

Pfleiderer, Georg 439

Philippson, Ludwig 78, 394-395, 397, 399402, 503-504, 543-544

Israelitisches Predigt- und Schul-Magazin 394

Jakob Tirado 504

Sepphoris und Rom 504

Philippson, Phöbus 394-395, 399-400

Die Marannen 394, 504

Die Vertreibung der Juden aus Spanien und Portugal 394

Picard, Jakob 164, 169, 179-180

Der Gezeichnete 182

Pick, Adolph 299

Pick, Otto 334, 336

Picquart, Marie-Georges 488

Pilatus, Pontius 38

Pinsker, Leon

Auto-Emanzipation 101

Pinski, Dovid 379

Das Erwachen 378

Eisik Scheftel 378, 382

Pinthus, Kurt 177, 181, 187, 284, 544

Jüdische Lyrik der Zeit 177

Piscator, Erwin 488
Pius XII., Papst 493

Piza, Josef 394

Platen-Hallermünde, August von 88-89, 449-

451

Der romantische Oedipus 90

Platon

Phaidon 49

Plessner, Helmut 560

Polański, Roman

The Pianist (Film) 570

Polenz, Wilhelm von

Der Büttnerbauer 28

Politzer, Heinz 204

Pollack, Ernst 334

Pollack, Oskar 327

Popovici, Traian 371

Poppelauer, M. (Verlag) 540, 542, 546, 548

Popper, William 515

Prawer, Siegbert Salomon 567-568

Between Two Worlds 567

Preisendanz, Wolfgang 86

Prinz, Joachim 170

Wir Juden 550

Quentin, Franz Siehe Strauß, Ludwig

Querido, Emanuel (Verlag) 402

Raabe, Wilhelm 37, 41

Der Hungerpastor 27

Die Chronik der Sperlingsgasse 28

Frau Salome 28

Holunderblüte 28

Rabenalt, Arthur Maria ... reitet für Deutschland (Film) 568

Rabener, Gottlieb Wilhelm 89

Rabinovici, Doron 261

Andernorts 261

Raddatz, Fritz J. 458

Radisch, Iris 457

Ralph Giordano

Die Bertinis 571

Ramler, Karl Wilhelm 54

Rapoport, Salomo Jehuda 463

Rappaport, Moritz 78

Rasch, Gustav

Pariser Spaziergänge 86

Raschi 9

Rathenau, Walter 207

Höre, Israel! 100 
Rauscher, Peter 12

Regel, Dirk

So ein Schlamassel (Film) 574

Rehfisch, Hans José

Die Affäre Dreyfus 488

Reich, Wilhelm 369

Reichmann, Eva G. 165

Reichwald, Annika 45-59

Reich-Ranicki, Marcel 31, 42, 457

Reinhardt, Max 484, 491-492

Reiss, Erich (Verlag) 170, 544, 550

Rëubeni, David 403

Reuss, Heinrich von 64

Rezzori (d'Arezzo), Gregor von 369

Ein Hermelin in Tschernopol 369

Ricoeur, Paul 305

Riemelt, Max 575

Rießer, Gabriel 503, 511

Rilke, Rainer Maria 179, 284, 369

Zwei Prager Geschichten 329

Rimbaud, Arthur 277

Rimon (Verlag) 548

Rinser, Luise

Jan Lobel aus Warschau 30

Rispart, Eugen 396, 518

Ritter, Karl GPU (Film) 568 Über alles in der Welt (Film) 568

Robert, Ludwig 480 Die Macht der Verhältnisse 480 Die Tochter Jephthas 481

Robespierre, Maximilien de 93

Rode, Walther (Rosenzweig) 369

Rodenberg, Julius 86

Die vierundzwanzig Stunden von Paris 86

Rödl, Josef 574

Schalom, meine Liebe (Film) 574

Roggenkamp, Viola

Familienleben 257

Rohling, August 300

Der Talmudjude 300

Rosegger, Peter 455

Rosenberg, Walter Siehe Vrba, Rudolf

Rosenkranz, Moses 225, 229, 368

Kindheit 362

Rosenthal, Fritz Siehe Ben-Chorin, Schalom

Rosenthal, Hugo 128

Rosenzweig, Franz 136, 191, 177-178, 191, 549

Stern der Erlösung 136, 146, 178
Roth, Joseph 141, 148, 276, 437, 439, 463-464

Autodafé des Geistes 147

Berliner Saisonbericht 456

Der Segen des ewigen Juden 147

Flucht ohne Ende 147

Hotel Savoy 147

Jedermann ohne Pass 147

Juden auf Wanderschaft 114, 147, 276

Radetzkymarsch 259-260

Rothgiesser, Fritz

Das Knabenschiff 182

Rothschild, Jakob 93

Rothschild, Thomas 498

Rousseau, Jean-Jacques 49, 427

Confessions 272

Rowohlt, Ernst (Verlag) 174

Rozenblit, Marsha 303-304

Rubaschow, Salman 377

Rubel, Maximilien 369

Rubel, Nomi 208

Rubiner, Ludwig 113, 144, 456

Rübner, Tuvia 214

Rudolf II. (Kaiser) 325, 341-342

Ruge, Arnold 90, 451

Rushdie, Salman 197

Rütten, Joseph 544, 549

Ruzowitzky, Stefan Die Fälscher (Film) 570

Saar, Ferdinand von 296-297, 299-300 Seligmann Hirsch 29, 296

Sabbatai Zwi 403

Sabel, Johannes 463-464, 467

Sacher-Masoch, Leopold von 28, 417 Besuch beim Wunderrabbi von Sadagora 366

Sachs, Michael 78

Sachs, Nelly 164, 167, 179, 193, 232-233, 245, 277, 436

Eli 445

In den Wohnungen des Todes 232

Sahl, Hans

Hausmusik 494-495

Salomon (Saaling), Rebecca 66

Salomon ben Josua Siehe Maimon, Salomon

Salomon, Gotthold 516

Salomon (König) 137

Salomon, Lea (Lilla) 65

Salten, Felix 112, 298, 306 
Salus, Hugo 330

Samson, Meta

Spatz macht sich 172-173

Samter, Max 164

Die Versuchung 172-173

Samuely, Nathan 418

Das Tüpferl auf dem i 441

Sandbank, Shimon 209

Saphir, Moritz Gottlieb 86-87, 89-90, 449452, 454

Deutsche humoristische Literatur 91

Sargent, Joseph und Mann, Daniel Playing for Time (Film) 570

Sarris, Andrew 566

Satanow, Isaak 52

Schachnowitz, Selig 401, 437, 442, 444

Flucht in die Heimat 172

Luftmenschen 442

Schächter, Solomon 16

Schadow, Johann Gottfried 63

Schalom, Shin 208

Schardt, Michael M. und Sudhoff, Dieter Prager deutsche Erzählungen 333

Schasar, Salman 117

Schenk, Otto 492

Schenker, Anatol 539

Scherbel, Fritz 546

Scherzer, Rosalie Beatrice Siehe Ausländer, Rose

Schick, Baruch 47

Schildt, Axel 196

Schiller, Friedrich 41, 479

Kabale und Liebe 182

Wilhelm Tell 491

Schindel, Robert 221, 260

Gebürtig 257-260

Schweigend ins Gespräch vertieft 228

Schindler, Liane Siehe Shmueli, Ilana

Schlegel, August Wilhelm 60, 88, 91, 449

Schlegel, Friedrich 60, 64

Schleiermacher, Charlotte 60

Schleiermacher, Friedrich Daniel Ernst 60-61, 63-64

Über die Religion 60

Versuch einer Theorie des geselligen

Betragens 60

Schlenstedt, Silvia 167

Schlomo ben Jizchak Siehe Raschi

Schlonsky. Abraham 208
Schmidt, Julian 455

Geschichte der deutschen Literatur im neunzehnten Jahrhundert 453

Schneider, Haim 213

Schneider, Lambert 549

Schneider, Michael 27

Schnitzler, Arthur 128, 298-303, 306, 310-315, 317, 319, 440

Der grüne Kakadu 306

Der Weg ins Freie 313-314

Liebelei 484

Lieutenant Gustl 310

Professor Bernhardi 311, 314, 484

Schochat, Asriel 45, 47

Schocken, Salman 540, 549-551

Scholem Alejchem 117, 375, 381, 419, 448, 547

Scholem, Arthur 546

Scholem, Gershom 13, 117, 209, 212, 377, 381382, 430, 438, 551

Von Berlin nach Jerusalem 431

Wider den Mythos vom deutsch-jüdischen

Gespräch 2

Scholem, Siegfried 546

Scholz, Wilhelm 87

Schönberg, Arnold 299

Moses und Aron 191-192

Schönerer, Georg Ritter von 301

Schönfels, Brigitte von 86

Schoor, Kerstin 164-188, 168

Schopenhauer, Arthur 287

Schorske, Carl E. 304

Schottländer, Benedict 78

Schreuder, Saskia 168, 539

Schüler, Meir 13

Schüler-Springorum, Stefanie 196

Schüller, Alexander 296-324, 325-361

Schulte, Christoph 45

Schulz, Bruno 279

Schulz, Georg-Michael 479-490

Schulze, Ursula 11

Schulze-Maizier, Friedrich 283

Begegnung mit Georg Heym 284

Schumsky, Dimitry 332

Schünzel, Reinhold 565, 567

Schwadron, Abraham 139

Mauschelpredigt eines Fanatikers 140

Schwarz, Johannes Valentin 515

Schwarz, Karl 547

Schwarz-Gardos, Alice 206, 213 
Scott, Walter 392-393, 395, 409

Ivanhoe 27

Sebald, W. G.

Austerlitz 31

Die Ausgewanderten 31

Seemann, Hermann 544-545

Seligmann, Barbara 261

Seligmann, Louis 393

Seligmann, Rafael 141, 246, 255-256, 377, 574

Bejahung und Verneinung des Galuth 140

Rubinsteins Versteigerung 245-246

Schalom, meine Liebe 574

Selle, Christian Gottlieb 63

Selpin, Herbert

Carl Peters (Film) 568

Seneca, Lucius Annaeus 38

Sessa, Karl Borromäus Alexander 26 Unser Verkehr 26, 480

Shaked, Gershon 435-436

Shakespeare, William 40, 91

Der Kaufmann von Venedig 301, 492- 494

König Lear 492

Shedletzky, Itta 10

Shmeruk, Chone 11

Shmueli, Ilana 212, 365, 368

Ein Kind aus guter Familie 368

Über mein Czernowitz erzählen (1924-

1944) 368

Zeitläufe 213

Zwischen dem Jetzt und dem Jetzt 213

Shveln (Verlag) 548

Sienerth, Stefan 370

Silbergleit, Arthur 117, 164

Der ewige Tag 180

Silbermann, Edith 368

Silburg, Mosche 384

Simmel, Georg 560-561

Der Fremde 560

Philosophie des Geldes 560

Simon, Ernst 479

Singer, Kurt 491

Singer, Ludwík 330

Sinsheimer, Hermann

Maria Nunnez 182, 402

Rabbi, Golem und Kaiser 402

Skolnik, Jonathan 473

Sokolow, Nahum 106

Sombart, Werner 455

Sommer, Ernst 355
Botschaft aus Granada 402

Die Revolte der Heiligen 355

Sommer, Maria 495

Soncino (Familie) 541

Sonnenthal, Adolf 306

Soskin, Selig Eugen 510

Spalding, Henry D. 448

Spangenberg, Max 283

Spanier, Dr. M[oritz] Leutnant Sender 159

Spector, Scott 332, 335

Speidel, Ludwig 86

Spektor, Mordechai 419

Spielberg, Steven

Schindler's List (Film) 570

Spindler, Karl

Der Jude 27

Spinoza, Baruch 50, 403

Spirek, Christiane Ida 334

Spitzer, Daniel 86, 298

Wiener Spaziergänge 298

Šrámek, Fráňa

Flammen 336

Staël, Anne Louise Germaine de De l'Allemagne 274

Stalin, Josef 247, 267

Staudinger, Barbara 12

Stauff, Philipp 127, 130 Die Juden in Literatur und Volk 130

Stein, Benjamin 253, 262 Die Leinwand 253

Stein, Leopold 396, 518

Steinbarg, Elieser 366

Steinecke, Hartmut 205

Steinhoff, Hans

Familientag im Hause Prellstein (Film) 565, 567

Steinhoff, Hans, Anton, Karl und Maisch, Herbert Ohm Krüger (Film) 568

Steinschneider, Moritz 70, 75-76, 396, 508

Die hebräischen Übersetzungen des

Mittelalters und die Juden als Dolmetscher 76

Jüdische Literatur 74, 138

Steinthal, Heymann 559

Stemmle, Robert Adolf

Die Affaire Blum (Film) 570

Stenzel, Abraham Nochem

Gideons Brandopfer 177 
Stern, David 466

Stern, Frank 572-573

Stern, Gerson 164, 201, 207-208, 436 Weg ohne Ende 171, 182, 402, 404

Stern, Günther Siegmund Siehe Anders, Günther

Sternbach, Hermann Wenn die Schakale feiern 158-159

Sternheim, Carl 485 Aus dem Bürgerlichen Heldenleben 485 Die Hose 485

Stettenheim, Julius 87,452

Stevens, George The Diary of Anne Frank (Film) 570

Stifter, Adalbert 41

Stoecker, Adolf 100

Stöhr, Ingo 334

Stomps, Victor Otto 180

Strauch, Gabriele 16

Strauß, Ludwig 80, 109, 117, 128-129, 164, 167, 208-209, 212-214, 382, 436

Der Tod des Verborgenen 445

Die zwiefache Heimat 214

Land Israel 180

Reichstreue und Volkstreue 113

Strittmatter, Thomas Viehjud Levi 573

Struck, Hermann 117, 275-276, 522

Sturmann, Manfred 164, 179

Bekenntnis 180

Herkunft und Gesinnung 180

Sudhoff, Dieter und Schardt, Michael M. Prager deutsche Erzählungen 333

Suhrkamp (Verlag) 552

Susmann, Margarete 558

Süß, Hermann 18

Süßkind von Trimberg 16

Sutzkever, Avrom

Die bleiernen Platten von Romm's

Druckerei 385

Szeps, Moriz 301

Szondi, Peter 224, 230, 559

Taaffe, Eduard Graf 300

Taberner, Stuart 572-573

Tábori, Cornelius 495

Tabori, George 236, 458, 495-497 Improvisationen über Shakespeares Shylock 496
Jubiläum 496-497

Kannibalen 495-497

Mein Kampf 236, 497-498

Mutters Courage 496

Tauber, Josef Samuel 414

Der Kadisch vor Kol Nidre 414

Der Retter 414

Die letzten Juden 414

Gawriel 414

Täubler, Eugen 547

Taussig, Nadja 203

Teller, Wilhelm 60

Telushkin, Joseph 448

Tendlau, Abraham 467, 472, 518

Das Buch der Sagen und Legenden Jüdischer Vorzeit 471

Theilhaber, Felix A. 110, 153 Jüdische Flieger im Kriege 158

Thieberger, Friedrich 521

Thiele, Martina 570

Tieck, Ludwig 91

Tietze, Hans 315

Timm, Erika 17, 19

Timms, Edward 305

Tisch, Ulrike 213

Tišma, Aleksandar

Das Buch Blam 222

Tismar, Jens 332

Toch, Michael 11

Toller, Ernst

Die Wandlung 157, 485

Torberg, Friedrich 208, 448

„Wai geschrien!“ oder Salcia Landmann ermordet den jüdischen Witz 448

Toulmin, Stephen 305

Toury, Jacob 502

Trakl, Georg 367

Treitschke, Heinrich von 283, 453, 455

Deutsche Geschichte im neunzehnten

Jahrhundert 453

Noch einige Bemerkungen zur Judenfrage

452

Unsere Aussichten 100

Treu, Abraham 396

Trietsch, Davis 116, 545, 547

Trojanow, Ilija 194

Trotta, Margarethe von Rosenstraße (Film) 572

Trotzki, Leo 487 
Tucholsky, Kurt 277, 449, 458

Tück, Jan-Heiner 440

Ucicky, Gustav

Heimkehr (Film) 568

Ungar, Hermann 332, 463-464

Der rote General 331, 487

Die Klasse 351

Die Verstümmelten 352-353

Geschichte eines Mordes 328

Unger, Erich 283, 285-287, 289

Politik und Metaphysik 286

Wedekinds ,In allen Wassern gewaschen' 288

Unzelmann, Friderike 65

Urban-Fahr, Susanne 539

Urzidil, Gertrude 209

Urzidil, Johannes 326

Da geht Kafka 326

Ussischkin, Menachem 106

Valéry, Paul 277

Van Hoddis, Jakob 283-284, 287

Von mir und vom Ich 286

Weltende 283-284

Varnhagen von Ense, Karl August 65-66, 89, 272

Varnhagen, Rahel Siehe Levin Varnhagen, Rahel

Veit, Brendel (Dorothea) 64

Veit, Moritz 542

Veit, Simon 64

Vergelis, Arn 385

Vergil 308

Verhaeren, Émile 288

Verlaine, Paul 369

Vertlib, Vladimir 264-265

Das besondere Gedächtnis der Rosa Masur 266, 278

Letzter Wunsch 265

Spiegel im fremden Wort 266

Zwischenstation 264-265, 278

Viertel, Berthold

Ostjuden 114

Vilsmaier, Joseph

Comedian Harmonists (Film) 572

Leo und Claire (Film) 573

Vital, Hajim

Peri Ez Hayyim 501
Vivanco, Pedro 303

Vogt-Maykopf, Chaim 3

Voigts, Manfred 122-134, 283-295

Voit, Friedrich 168

Völpel, Annegret 539

Voltaire 40, 273

Von Arnstein, Fanny 66

Von Brinkmann, Gustav 65

Von Dohna, Alexander 64

Von Eskeles, Cäcilie 66

Voß, Christian Friedrich 541

Voß, Johann Heinrich 91

Voß, Julius von 26

Der travestirte Nathan der Weise 26, 480

Euer Verkehr 480

Vrba, Rudolf und Wetzler, Alfred

Bericht der Auschwitzflüchtlinge (Ende April 1944) 228

Vrba, Rudolf 227-228

Die mißachtete Warnung 228

I cannot forgive 228

Vrchlický, Jaroslav 336

Wagenseil, Johann Christoph

Artushof 18

Wagner, Richard 108, 111, 455, 485

Das Judenthum in der Musik 29, 84, 99

Mein Leben 453

Wagner-Egelhaaf, Martina 422

Waldinger, Ernst

In Memoriam Leutant Beer 160

Waldoks, Moshe 448

Wallas, Armin A. 210, 306, 443

Walser, Martin 24, 41, 244

Tod eines Kritikers 31, 42

Walter, Hans-Albert 195

Warburg, Aby 560

Warburg, Otto 510

Warnock, Robert G. 17

Waschneck, Erich

Die Rothschilds (Film) 568

Wassermann, Henry 165

Wassermann, Jakob 118, 255, 302, 440

Der Fall Maurizius 441

Die Juden von Zirndorf 402-403

Mein Weg als Deutscher und Jude 117, 429-430

Wedekind, Frank 284, 288

Rabbi Esra 289 
Weidner, Daniel 555-564

Weil, Jacob 463, 467-469, 471-472, 475

Fragmente aus dem Talmud und den Rabbinen 467

Weil, Robert

Schulaufsätze des Poldi Huber 546

Weil, Simone 432

Weill, Alexandre 418

Weininger, Otto 298-299, 305, 313, 353, 449

Geschlecht und Charakter 313

Weinzierl, Ulrich 457

Weisel, Leopold 397

Weiß, Ernst 275, 277, 339

Der Kampf Siehe Franziska

Franziska 338

Weiss, Ilse 179

Weiss, Peter 194, 196, 227, 245

Die Ästhetik des Widerstands 194, 227

Die Ermittlung 227, 493

Weissberg, Liliane 60-69, 273

Weißglas, Immanuel 229, 368

Weizmann, Chaim 106

Weltsch, Felix 111, 208, 335, 343, 521

Weltsch, Robert 334, 509

Wenzel, Edith 16

Werfel, Franz 112-113, 118, 275, 334, 353, 437, 439, 544

Der Abituriententag 353

Der Spiegelmensch 484

Der Weltfreund 337

Die Troerinnen 484

Paulus unter den Juden 487

Werner, Klaus 369-370

Wertheimer, Martha 164

Wertheimer, Samson 12

Wessel, Kai und Kleinert, Andreas

Klemperer - Ein Leben in Deutschland

(Film) 573

Wessely, Hartwig 52

Wessely, Naphtali Herz 47, 51

Divrej schalom we-emet 47

Gan Na'ul 47

Westermann, Klaus 456

Wetzlar, Isaak 47

Wetzler, Alfred 227-228

Wetzler, Alfred und Vrba, Rudolf

Bericht der Auschwitzflüchtlinge (Ende

April 1944) 228

Wieland, Christoph Martin 89
Wienbarg, Ludolf 94

Wiener, Oskar 330, 336

Wiesel, Pauline 65

Wild, Dölf 15

Wilhelm II. (Kaiser) 130, 457

Burgfriedensrede 153

Wilhelm, Carl

Der Stolz der Firma (Film) 566

Die Firma heiratet (Film) 566

Wilhelmy, Petra 62

Wilkomirski, Binjamin 223

Bruchstücke 256

Williams, Raymond 205

Winder, Ludwig 330, 335, 353-354, 436

Die jüdische Orgel 353

Geschichte meines Vaters 328

Hugo 354

Turnlehrer Pravda 354

Winder, Maximilian 330, 353

Winkler, Manfred 213-214

Winter, Jakob und Wünsche, August

Die jüdische Literatur seit dem Abschluss des Canons 79

Wirnt von Grafenberg

Artushof 15

Wirtz, Michaela 565-579

Wisten, Fritz 492

Wistrich, Robert S. 303

Witte, Bernd 474-475

Wittgenstein, Ludwig 305

Tractatus 305

Wohl von Haselberg, Lea 574

Wohl, Jeanette 94

Wohlgemuth, Joseph 440, 506

Wolf, Friedrich August 71

Wolf, Friedrich

Cyankali 487

Wolf, Immanuel 70-73

Über den Begriff einer Wissenschaft des Judenthums 71

Wolf, Joseph 502

Wolf, Konrad Ich war 19 (Film) 571

Wolfenstein, Alfred 112, 118, 141, 208

Jüdisches Wesen und neue Dichtung 142

Wolff, Alfred 23

Wolff, Bernhard 86

Wolff, Christian 51

Wolff, Kurt (Verlag) 544, 549 
Wolff, Theodor 87

Wolfskehl, Karl 110, 141, 148, 558

Die Stimme spricht 148, 179

Hiob 192

Wolpe, Stephan 208

Woltmann, Karl Ludwig von 64

Wonnenberg, Felice Naomi 573

Worlik, Emanuel

Der Fahnenreiter von Prag 402

Wronkow, Ludwig 117

Wünsche, August und Winter, Jakob

Die jüdische Literatur seit dem Abschluss des Canons 79

Wüst, Hans Werner

Massel braucht der Mensch 448

Xiangcheng, Zhu 367

Yourgrau, Wolfgang 204

Zadek, Peter 493-494, 498

My Way 494

Zangwill, Israel 418

Zarncke, Friedrich 15

Zech, Paul 288

Zedner, Josef 467, 469-470, 473

Auswahl historischer Stücke aus

hebräischen Schriftstellern 469

Zerlett, Hans $\mathrm{H}$.

Robert und Bertram (Film) 568

Venus vor Gericht (Film) 568

Zibaso, Magali 213

Ziesel, Kurt

Daniel in der Löwengrube 30

Und was bleibt, ist der Mensch 30

Zimmermann, Hans Dieter 337

Zinner, Hedda 494

Ravensbrücker Ballade 494

Zivier, Georg 164

Zlocisti, Theodor 105, 110, 521, 547

Zohn, Harry 306

Zola, Émile 488

Zuckermann, Hugo 154

An Judas Jugend 154

Gedichte 115

Lied ans Maschinengewehr 154
Makkabäer 5675115

Österreichisches Reiterlied 154

Psalm 154

Zuckmayer, Carl 141

Des Teufels General 492

Weltbürgertum 148

Zunz, Leopold 70-75, 79, 138, 463, 502-503, 506,516

Die jüdische Literatur 2, 73

Zweig, Arnold 117-118, 151, 204, 208, 211, 275, 303, 479, 485, 547

Abigail und Nabal 485

Allenfalls unter ,Antworten` zu bringen?

160

Bilanz der deutschen Judenheit 165

Das Mittel des Geistes 113

Das ostjüdische Antlitz 114

Der große Krieg der weißen Männer 160

Die Sendung Semaels 486

Die Umkehr Siehe Die Umkehr des

Abtrünnigen

Die Umkehr des Abtrünnigen 486

Erziehung vor Verdun 160

Juden auf der deutschen Bühne 486

Judenzählung vor Verdun 156-157

Junge Frau von 1914160

Ritualmord in Ungarn Siehe Die Sendung

Semaels

Zweig, Max 211

Davidia 211

Die Marranen 211

Zweig, Stefan 141, 145, 193, 298, 302, 304, 307-308, 312, 315-316, 318-319

Buchmendel 316

Das neue Pathos 288

Der begrabene Leuchter 318

Die Welt von Gestern 148

Erasmus von Rotterdam 182

Im Schnee 316

Jeremias 191-193, 307, 318, 440, 486

Rachel rechtet mit Gott 307

Zwerenz, Gerhard

Die Erde ist unbewohnbar wie der Mond 31 